\title{
Die Familie von Lobkowicz als Vermittler der kulturellen Wechselwirkungen im böhmisch-sächsischen Grenzraum um 1500
}

\author{
von \\ PETR HLAVÁČEK
}

Seit einigen Jahrzehnten florieren in Europa sowie in Amerika die sog. borderlands studies, d. h. eine „Anthropologie“ von Grenzregionen, die deutlich zeigen, dass ein historisches Grenzland nicht automatisch eine politische oder kulturelle Peripherie war. Diese Prämisse ist gerade für das sächsisch-böhmische Erzgebirge als eine historische Kulturlandschaft gültig, die keinesfalls eine künstliche Konstruktion der modernen Historiografie ist. Im gesamten Mittelalter verlief im breiteren sächsisch-böhmischen respektive meißnisch-böhmischen Grenzraum ein vielschichtiger Prozess, der durch politische, religiöse, kulturelle oder wirtschaftliche Interaktionen zu einer sehr dynamischen Vernetzung führte. Dieser Prozess fand seinen Höhepunkt im 16. Jahrhundert, in der Zeit der großen Bergbaukonjunktur und der Wittenberger Reformation, als sich auch im böhmischen Erzgebirge der größte Teil der Bevölkerung zur „Confessio Augustana“ bekannte. Ebenfalls für die böhmischen sowie sächsischen Intellektuellen waren die „Sudeta Montes“ eine wichtige Region der europäischen humanistischen Topografie, überdies mit einer sehr spezifischen regionalen Identität. Die komplexe Durchlässigkeit der sächsisch-böhmischen Grenze endete im 17. Jahrhundert mit der endgültigen Konfessionalisierung auf beiden Grenzseiten. ${ }^{1}$

$\mathrm{Zu}$ den wichtigsten Akteuren des intensiven Kulturtransfers in der sächsisch-böhmischen Grenzregion gehörten auch die hiesigen Adelsfamilien, die oft zwischen der böhmischen oder sächsischen Landesidentität oszillierten. Eine Schlüsselrolle spielte in diesem Sinne die Familie der Hassensteiner von Lobkowicz (Hasištejnští z Lobkowicz). Sie engagierte sich in Nordwestböhmen seit 1418, als die Burg Hassenstein (Hasištejn) bei Kaaden (Kadaň) zu ihrer Residenz wurde. 1459 wurden die Mitglieder der Familie von Kaiser Friedrich III. in den Reichsfreiherrenstand erhoben, erst 1479 wurden sie in den böhmischen Herrenstand aufgenommen. Zu ihrer Herrschaft Has-

1 Petr HlaváČeK, Der böhmisch-sächsische Grenzraum im Spätmittelalter und in der Frühneuzeit zwischen Integration und Desintegration, in: Miloš Řezník (Hg.), Grenzraum und Transfer. Perspektiven der Geschichtswissenschaft in Sachsen und Tschechien (Chemnitzer Europastudien 5), Berlin 2007, S. 83-94; Ders., Catholics, Utraquists and Lutherans in Northwestern Bohemia, or Public Space as a Medium for Declaring Confessional Identity, in: Milena Bartlová/Michal Šroněk (Hg.), Public Communication in European Reformation. Artistic and other Media in Central Europe 1380-1620, Prague 2007, S. 279-297; Ders., Die Christenheit oder Europa. Zu konfessionell-geographischen Vorstellungen im Böhmen des 15. und 16. Jahrhunderts, in: Evelin Wetter (Hg.), Formierungen des konfessionellen Raumes in Ostmitteleuropa (Forschungen zur Geschichte und Kultur des östlichen Mitteleuropa 33), Stuttgart 2008, S. 319-331; Ders., Die Eliten Nordwestböhmens in der kirchenpolitischen und theologischen Interaktion mit sächsischen Universitäten der Reformationszeit, in: Acta Universitatis Carolinae - Historia Universitatis Carolinae Pragensis 49 (2009), S. 151-154. 
senstein-Pressnitz (Hasištejn-Př́sečnice) gehörte lange Zeit auch das sächsische Städtchen Schlettau mit seiner Umgebung, das einen Bestandteil des Kaadner Dekanats im Prager Erzbistum bildete. ${ }^{2}$

1469 verpfändete der böhmische König Georg von Podiebrad die freie königliche Stadt Kaaden an der Eger, damals eine der wichtigsten Städte Nordwestböhmens, an die Familie der Hassensteiner von Lobkowicz. Gleichzeitig wurde Johannes Hassensteiner von Lobkowicz zum königlichen Hauptmann der Stadt ernannt. Die Stadt Kaaden wurde mit der Kaadner Burg zur lobkowiczschen Hauptresidenz, und zwar bis zum Jahre 1519. ${ }^{3}$ Durch eine Entscheidung des böhmischen Königs Wladislaus Jagello hielten die Brüder Johannes, Bohuslaus, Jaroslaus I. und Nikolaus III. Hassensteiner von Lobkowicz seit 1481 auch die sog. Fundatorenrechte des Franziskanerklosters der Vierzehn heiligen Nothelfer in Kaaden. Dieses Kloster, das sich zur wichtigsten Begräbnisstätte der Familie von Lobkowicz entwickelte, stand in enger Verbindung mit den zwei gleichnamigen Wallfahrtsorten, also mit Vierzehnheiligen bei Bamberg in Oberfranken und Vierzehnheiligen bei Jena in Thüringen. Gerade der Kult der Vierzehn heiligen Nothelfer stellt ein Beispiel eines konkreten Kulturtransfers zwischen Franken, Thüringen, respektive Sachsen und Böhmen, dar, wobei in diesem Prozess die thüringische Familie Vitzthum eine sehr wichtige Rolle spielte. Das Adelsgeschlecht ließ sich nämlich nach dem sächsischen Bruderkrieg in Böhmen nieder, direkt im Kaadner Land, und wurde zum Nachbarn und Rivalen der Familie von Lobkowicz. In den lobkowiczschen Zeiten blühte in Kaaden auch eine jüdische Gemeinde, deren Mitglieder nicht in einem Ghetto lebten, sondern vollberechtigte Kaadner Bürger waren. ${ }^{4}$

Eine große Bedeutung für die kulturellen böhmisch-sächsischen Kontakte um 1500 hatte Bohuslaus Hassensteiner von Lobkowicz, ein humanistischer Literat mit einer gesamteuropäischen Perspektive. Zwischen 1475 und 1482 studierte er in Italien an den Universitäten in Bologna und Ferrara. Später unternahm er in den Jahren 1490 und 1491 eine einzigartige Pilgerfahrt mit humanistisch orientierten Interessen, er besuchte

2 Eine gute Synthese ist immer: FrIEdrich Bernau, Hassenstein. Ein Beitrag zur Geschichte des Erzgebirgs, Böhmisch Leipa 1893. Vgl. Petr Hlaváčex, Zu konfessionellen Beziehungen im böhmisch-sächsischen Erzgebirge im Reformationszeitalter, in: Bernd Stephan/Martin Lange (Hg.), Wortwechsel. Das Kolloquium zum 475. Geburtstag der Evangelisch-Lutherischen Landeskirche Sachsens in Annaberg-Buchholz, Annaberg-Buchholz 2015, S. 62-73.

3 Petr HlaváčeK, Církevně-politický a spirituální profil Jana Hasištejnského z Lobkowicz [Das kirchenpolitische und spirituelle Profil des Johannes Hassensteiner von Lobkowicz], in: Michaela Ottová/Aleš Mudra (Hg.), Mýtus Ulrich Creutz. Vizuální kultura v Kadani za Jana Hasištejnského z Lobkowicz (1469-1517), Litoměřice/Praha 2017, S. 48-53.

4 Ders., Zur christozentrischen Dimension des spätmittelalterlichen Kultes der Vierzehn heiligen Nothelfer, in: Jiří Fajt/Markus Hörsch (Hg.), Künstlerische Wechselwirkungen in Mitteleuropa (Studia Jagellonica Lipsiensia 1), Ostfildern 2006, S. 405-426; Ders., Die kirchenpolitischen Aspekte des Kultes der Vierzehn heiligen Nothelfer im sächsisch-böhmischen Raum im ausgehenden Mittelalter, in: André Thieme/Uwe Tresp/Birgit Finger (Hg.), Eger 1459. Fürstentreffen zwischen Sachsen, Böhmen und ihren Nachbarn (Saxonia 13), Wettin-Löbejün 2011, S. 383-389. Allgemein zur Verehrung der Nothelfer im spätmittelalterlichen Europa und besonders im böhmischsächsischen Raum: Petr HLaváček, Čtrnáct svatých Pomocníků. K pozdně středověké spiritualitě elit a její christocentrické dimenzi [Die Vierzehn heiligen Nothelfer. Zur spätmittelalterlichen Elitenspiritualität und ihrer christozentrischen Dimension], Praha 2014. 


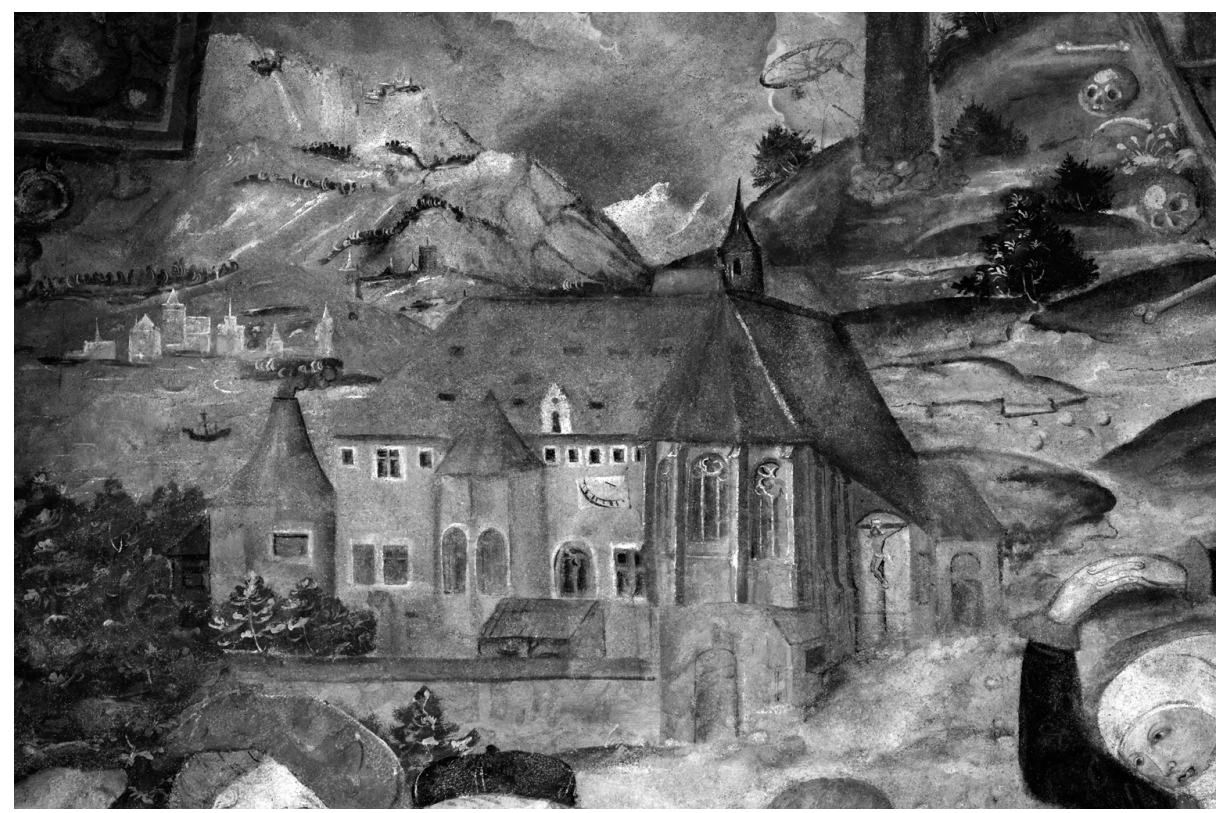

Abb. 1: Das Franziskanerkloster in Kaaden (Kadaň), Wandmalerei im Presbyterium der Kirche der Vierzebn heiligen Nothelfer, um 1530.

das Heilige Land mit Jerusalem und auch viele antike Denkmäler in Syrien, Ägypten, Kleinasien und Griechenland. Bohuslaus wirkte dann einige Jahre als Höfling des Königs Wladislaus Jagello in Ofen (Buda), aber seine Zuflucht fand er zu Hause, auf der Burg Hassenstein bei Kaaden am Fuß des Erzgebirges. Hier leitete er bis zu seinem Tod im Jahre 1510 eine eigene, sehr berühmte humanistische Akademie, an der Edelleute und Bürger, Katholiken und Utraquisten, Priester und Laien, sowohl Tschechen als auch Deutsche studierten. Als Pädagoge war hier auch der thüringische Dichter Johannes Sturnus von Schmalkalden tätig, ein Freund von Conrad Celtis und Mitglied der „Sodalitas litteraria Danubiana“. Zu dieser humanistischen Gesellschaft gehörten ebenfalls der Narr Georg („Jiř́k“) und der getaufte Mohr Martin, der aus Nordafrika nach Böhmen gekommen war. Ihre Bildung gewannen auf Hassenstein auch Bohuslaus' Neffen Sigismund und Nikolaus, ebenso auch Wolfgang von Kaaden, ein unehelicher Sohn von Johannes Hassensteiner von Lobkowicz. Den Studenten standen hier nicht nur eine ausgezeichnete humanistische Bibliothek aller Fächer zur Verfügung, sondern auch ein Kabinett mit Weltkarten und Globen sowie ein astronomisches Observatorium im Burgturm.

Bohuslaus Hassensteiner von Lobkowicz korrespondierte regelmäßig mit vielen europäischen Gelehrten vom Rheinland bis Ungarn, von Sachsen bis Italien. In eigenen Briefen, Gedichten und anderen Texten reflektierte er die Probleme der ganzen Welt und interessierte sich lebhaft auch für das „neuentdeckte“ Amerika. Aber es waren gerade die "Saxones“, seine unmittelbaren Nachbarn, mit denen Bohuslaus wirklich intensiv kommunizierte. $\mathrm{Zu}$ seinen humanistischen Freunden gehörten zum Beispiel Paul Morgenstern, Absolvent der Leipziger Universität und Abt des Zisterzienserklosters Grünhain im sächsischen Erzgebirge, weiterhin der süddeutsche Humanist Konrad Wimpina, ehemals Universitätsprofessor in Leipzig, oder Martin Pollich, Arzt, Philosoph und Astrologe sowie Gründungsrektor der Universität 
Wittenberg. Vielleicht wurde Bohuslaus deshalb zum Kopf der Wittenberger humanistischen Gesellschaft „Sodalitas Leucopolitana“ gewählt. Auf den Leipziger Messen besorgten Bohuslaus' Agenten neue Bücher, und junge Männer aus dem KaadnerHassensteiner humanistischen Kreis gingen an die Universitäten in Leipzig und Wittenberg. An der Wittenberger Hochschule studierten beispielsweise Bohuslaus' Neffe Sigismund Hassensteiner von Lobkowicz oder Matthäus Aurogallus, der spätere Professor für Hebraistik und sogar Rektor der Universität zu Wittenberg. Beide hielten immer gute Kontakte zur böhmischen Seite des Erzgebirges, und sie unterstützten auch die Verbreitung der Wittenberger Reformation im nordwestböhmischen Gebiet. Aurogallus war Luthers Freund und sein Mitarbeiter bei der Bibelübersetzung. Die Bibliothek auf der Burg Hassenstein bei Kaaden erweckte die Aufmerksamkeit der sächsischen Humanisten und Kirchenreformatoren. So suchte um 1520 Joachim Camerarius, später ein berühmter Philologe und Hebraist, die Burg Hassenstein auf, um hier eine wertvolle griechische Handschrift zu finden. Mit Hilfe von Aurogallus liehen sich Luther und Melanchthon etwa 70 Bücher nach Wittenberg aus. Noch 1553 bewunderte der französische Humanist und Hugenotte Hubert Languet, später ein sächsischer Diplomat, die außergewöhnliche Hassensteiner Bibliothek. ${ }^{5}$

Erhebliche politische und damit auch kulturelle Verbindungen über die böhmischsächsische Landesgrenze pflegte Bohuslaus' älterer Bruder Johannes Hassensteiner von Lobkowicz, ein tschechisch schreibender Literat und königlicher Diplomat, der zum Beispiel in Italien und Burgund weilte. Seine Residenzstadt Kaaden mit der Kaadner Burg bildeten ein lebendiges Kultur- und Kunstzentrum. Schon 1476 empfahl er dem jungen böhmischen König Wladislaus Jagello für die Erneuerung der Prager Burg einen in Nordwestböhmen sowie in Sachsen gut bekannten Baumeister: Erhard Bauer von Eichstätt. Viele exzellente Künstler wirkten ebenfalls im Auftrag von Johannes Hassensteiner von Lobkowicz in der Franziskanerkirche in Kaaden, welche zu einem spirituellen Zentrum der ganzen Familie wurde.

Eine konstitutive Bedeutung für die persönliche, christozentrisch orientierte Frömmigkeit des Kaadner Pfandherrn Johannes hatte 1493 seine Pilgerreise ins Heilige Land. Seiner Führung vertraute sich in Venedig die große Wallfahrtsgruppe des bayerischen Herzogs Christoph des Starken und des sächsischen Kurfürsten Friedrich des Weisen an. Gerade mit Herzog Christoph besuchte Johannes die prunkvollen Kirchen, und sie kauften gemeinsam Luxusgüter. Damals lernte er auch den Maler Lucas Cranach den Älteren kennen, der nach dem Tod des bayerischen Herrschers auf der Insel Rhodos in den Dienst des Kurfürsten von Sachsen trat. ${ }^{6}$ Seit 1493 können wir von einem ständigen Kontakt des böhmischen Aristokraten Johannes Hassensteiner von Lobkowicz zum Kurfürsten Friedrich den Weisen sprechen. Johannes begegnete dem Kurfürsten beispielsweise 1495 in Wittenberg, wie wir aus der Korrespondenz seines Bruders Bohuslaus wissen. Und schon 1496 schickte Johannes dem Kurfürsten seinen Baumeister Jorgen von Kaaden nach Torgau, um die dortige steinerne Brücke zu vollenden. Es war vielleicht gerade Jorgen von Kaaden, der dann die neue Bautechnik der sog. Zellengewölbe aus Sachsen nach Böhmen transferierte. Diese Innovation wurde

5 Zu Bohuslaus' Biografie vgl. z. B. Bohuslaus Hassensteinius a LobKowicz, Opera poetica (Bibliotheca scriptorum graecorum et romanorum Teubneriana), hrsg. von Marta Vaculínová, München/Leipzig 2006; Kamil Boldan/Emma URbánKovÁ, Rekonstrukce knihovny Bohuslava Hasištejnského z Lobkovic [Die Rekonstruktion der Bibliothek des Bohuslaus Hassensteiner von Lobkowicz], Praha 2009.

6 Werner Paravicini (Hg.), Europäische Reiseberichte des späten Mittelalters. Eine analytische Bibliographie, Teil 1: Deutsche Reiseberichte, bearb. von Christian Halm (Kieler Werkstücke D/5), Frankfurt am Main u. a. 1994, S. 244 f. 
mehrmals auch in der lobkowiczschen Residenzstadt Kaaden genutzt, zuerst in den lobkowiczschen Räumen des Franziskanerklosters, später zum Beispiel auf der Kaadner Burg. Der wichtigste Beleg des Architekturtransfers der meißnischen Spätgotik mit ihren Zellengewölben ist in Kaaden die Imitation des Jerusalemer Coenaculums, d. h. des angeblichen Abendmahlsaals Jesu, im ersten Geschoss des Kaadner Franziskanerklosters. Gerade hier traf sich eine lobkowiczsche Reformgruppe, eine gewisse Bruderschaft des Heiligen Geistes. ${ }^{7}$

Die Familie von Lobkowicz verwandelte die ihr verpfändete königliche Stadt Kaaden an der Wende vom Mittelalter zur Frühen Neuzeit in einen dynamischen Brennpunkt der kulturellen böhmisch-sächsischen Wechselwirkungen. Nach Kaaden kamen aus den Ländern der sächsischen Kurfürsten und Herzöge zum Beispiel der franziskanische Theologe Eberhard Ablauf, der Maler Johannes von Plauen, der Baumeister Jacob Haylmann oder der Bildhauer Ulrich Creutz, welcher die sehr ausdrucksvoll gestaltete Tumba des Johannes Hassensteiner von Lobkowicz in der Franziskanerkirche in Kaaden schuf. Die Tafelbilder für die neue Kirche in der jungen Bergstadt Annaberg im sächsischen Erzgebirge fertigte auf der anderen Seite zwischen 1515 und 1524 der Maler Hans Frank aus Kaaden. Interessant ist auch, dass die Bürgerfamilien aus Kaaden und Annaberg viele Verwandtschaftsbeziehungen verbanden. Die Kaadner unterstützten nicht nur maßgeblich den Aufbau der Annaberger Pfarrkirche, sondern auch die Errichtung der dortigen Franziskanerkirche. ${ }^{8}$

Das bedeutendste und exklusive Beispiel des kulturellen sächsischen Einflusses auf die Familie der Hassensteiner von Lobkowicz sind die Cranachschen Wandmalereien in der schon mehrmals erwähnten Franziskanerkirche der Vierzehn heiligen Nothelfer in Kaaden. Es geht dabei vor allem um die prachtvollen Gemälde im Presbyterium, die zwischen 1520 und 1530 im Auftrag des Herrn Jaroslaus Hassensteiner von Lobkowicz, Johannes' Sohn, und seiner Gemahlin Margaretha von Plauen entstanden. Die Wandmalerei zeichnete sich durch vielfache ikonografische Allegorien aus, besonders die Darstellungen der Kreuzigung und Beweinung Christi und auch die doppelte Donatorenszene mit der Familie von Lobkowicz. Bemerkenswert scheint vor allem die Szene mit Jaroslaus Hassensteiner von Lobkowicz und seinen Söhnen Heinrich und Sebastian in einer Konversation mit den heiligen Jungfrauen Katharina und Barbara zu sein, oder auch die zweite Szene mit Margaretha von Plauen vor der Jungfrau Maria mit dem Jesuskind.

Besondere Aufmerksamkeit erwecken ebenfalls die realistischen Abbildungen des Franziskanerklosters in Kaaden oder der Burg Hassenstein, die mit ihrer Architektur zugleich eine Darstellung des biblischen Jerusalem sind, was früher auch das Ziel der Pilgerreise von Bohuslaus und Johannes Hassensteiner von Lobkowicz war. Die bib-

7 Vgl. Petr Hlaváček, Kadaň mezi středověkem a novověkem. Deset kapitol z kulturních a náboženských dějin severozápadních Čech [Kaaden zwischen Mittelalter und Neuzeit. Zehn Kapitel aus der Kultur- und Kirchengeschichte Nordwestböhmens], Ústí nad Labem 2005, S. 87-169; Ders., Die böhmischen Franziskaner im ausgehenden Mittelalter. Studien zur Kirchen- und Kulturgeschichte Ostmitteleuropas (Forschungen zur Geschichte und Kultur des östlichen Mitteleuropa 40), Stuttgart 2011, S. 31-38, 78 81, 127-134, 164-169.

8 Dazu allgemein: Petr HlaváčEk, Nový Jeruzalém? Př́iběh františkánského kláštera Čtrnácti sv. Pomocníků v Kadani [Das Neue Jerusalem? Die Geschichte des Klosters der Vierzehn heiligen Nothelfer in Kaaden], Kadaň 2013. Vgl. Ders., Eberhard Ablauff de Rheno $(\dagger$ 1528) im Geistesleben der böhmischen Franziskaner am Anfang der Frühen Neuzeit, in: Heidemarie Specht/Tomáš Černušák (Hg.), Leben und Alltag in böhmisch-mährischen und niederösterreichischen Klöstern in Spätmittelalter und Neuzeit (Monastica Historia 1), St. Pölten/Brno 2011, S. 136-146. 
lische Topografie ist hier mit realen Orten um Kaaden vermischt. In den einzelnen Darstellungen findet sich auch eine markante ikonografische Ambivalenz, denn die hier abgebildeten biblischen Akteure in den christologischen Szenen sind gleichzeitig die Porträts der Familie Lobkowicz. Der heilige Josef von Arimathia in der Kreuzigungsszene hat das Antlitz des alternden Johannes Hassensteiner von Lobkowicz diese Identifikation ist auch durch eine vielsagende Geste zwischen Josef oder Johannes und dem heiligen Evangelisten Johannes, seinem Namenspatron, naheliegend. Wiederum erscheint unser Johannes als Josef von Arimathia in der Beweinungsszene, überdies in einer Konversation mit dem heiligen Nikodemus, dessen Züge mit dem benachbarten Porträt des Jaroslaus Hassensteiner von Lobkowicz identisch sind. Josef von Arimathia neigt sich zu Nikodemus, als ob Vater Johannes seinen Sohn Jaroslaus über die Geheimnisse des Todes und der Auferstehung Christi belehrt. Zusätzlich ist die danebenstehende weibliche Person, vermutlich die „altera Maria“ aus dem Evangelium nach Matthäus, gleichzeitig ein Porträt von Jaroslaus' Gemahlin Margaretha von Plauen. Die abgebildeten Mitglieder der Familie von Lobkowicz sind hier also mit dem nahestehenden Umkreis Jesu identifiziert und bekannten sich damit ostentativ zur eigenen christozentrischen Spiritualität. ${ }^{9}$

Doch wer war eigentlich Margaretha von Plauen, diese einflussreiche Frau aus dem Geschlecht der Hassensteiner von Lobkowicz? Ihr Vater Heinrich III. von Plauen ( $\dagger$ 1519) übte das Burggrafenamt in Meißen aus, seit 1482 war er auch der Landvogt der Niederlausitz, die damals zur Böhmischen Krone gehörte. Margarethas Mutter war Barbara von Anhalt (†1532/33). Die Herren von Plauen hielten als Meißner Burggrafen auch die Fundatorenrechte des Klarissenklosters in Seußlitz bei Meißen, und sie pflegten vielfältige Kontakte mit anderen franziskanischen Institutionen in den sächsischen Ländern. Margarethas Bruder Heinrich IV. von Plauen $(† 1554)$ diente als Kanzler des böhmischen Königs Ferdinand I. von Habsburg. In seinen Herrschaften im Vogtland hielt er sich jedoch nicht auf, denn sie wurden von dem ernestinischen (lutherischen) Zweig des sächsischen Hauses Wettin besetzt. Die Ehebünde zwischen den Familien von Lobkowicz und von Plauen bildeten keine Ausnahme. Beispielsweise wurde Jaroslaus' Cousin Nikolaus IV. Hassensteiner von Lobkowicz $(† 1531)$ mit Brigitta von Plauen $(\dagger 1552)$ verheiratet. ${ }^{10}$

Gute Verbindungen mit Sachsen pflegten auch die Kaadner Franziskaner, besonders zu ihren Mitbrüdern in Annaberg. Das Annaberger Kloster wurde 1502 von dem erasmianisch orientierten Herzog Georg von Sachsen gegründet, dem Sohn der Zdena von Böhmen († 1510), der Tochter des böhmischen „Ketzerkönigs“ Georg von Podiebrad. Später kam es unter den Franziskanern in Annaberg wegen Luthers Lehre zur Spaltung, und ein Teil der hiesigen Brüder fand 1540 ihre Zuflucht gerade in Kaaden. ${ }^{11}$

9 Petr Hlaváček, Čtrnáct svatých Pomocníků (wie Anm. 4), S. 218-250. Aktuell: Jan RoYT, Nástěnné malby v chóru klášterního kostela [Die Wandmalereien im Chor der Klosterkirche], in: Ottová/Mudra, Mýtus Ulrich Creutz (wie Anm. 3), S. 96-104.

10 Johannes Richter, Burggraf Heinrich IV. von Meissen, Graf zu Hartenstein, Herr zu Plauen und Gera - „Der Eroberer von Hof“, in: Geschichte am Obermain 19 (1993/94), S. 47-55; Gerhard Billig, Die Burggrafen von Meißen aus dem Hause Plauen - ein Nachspiel zur reichsunmittelbaren Stellung und Herrschaft der Vögte von Weida, Plauen und Gera, Teil 2, in: Mitteilungen des Vereins für Vogtländische Geschichte, Volks- und Landeskunde N. F. 6 (1998), S. 51-82.

11 Sven Rabeler/Alexandra Kursawe/Claudia Ulrich (Hg.), Briefe der Herzogin Sidonia von Sachsen (1449-1510) an ihren Sohn Georg (1471-1539) (Mitteilungen der Residenz-Kommission der Akademie der Wissenschaften zu Göttingen, Sonderheft 


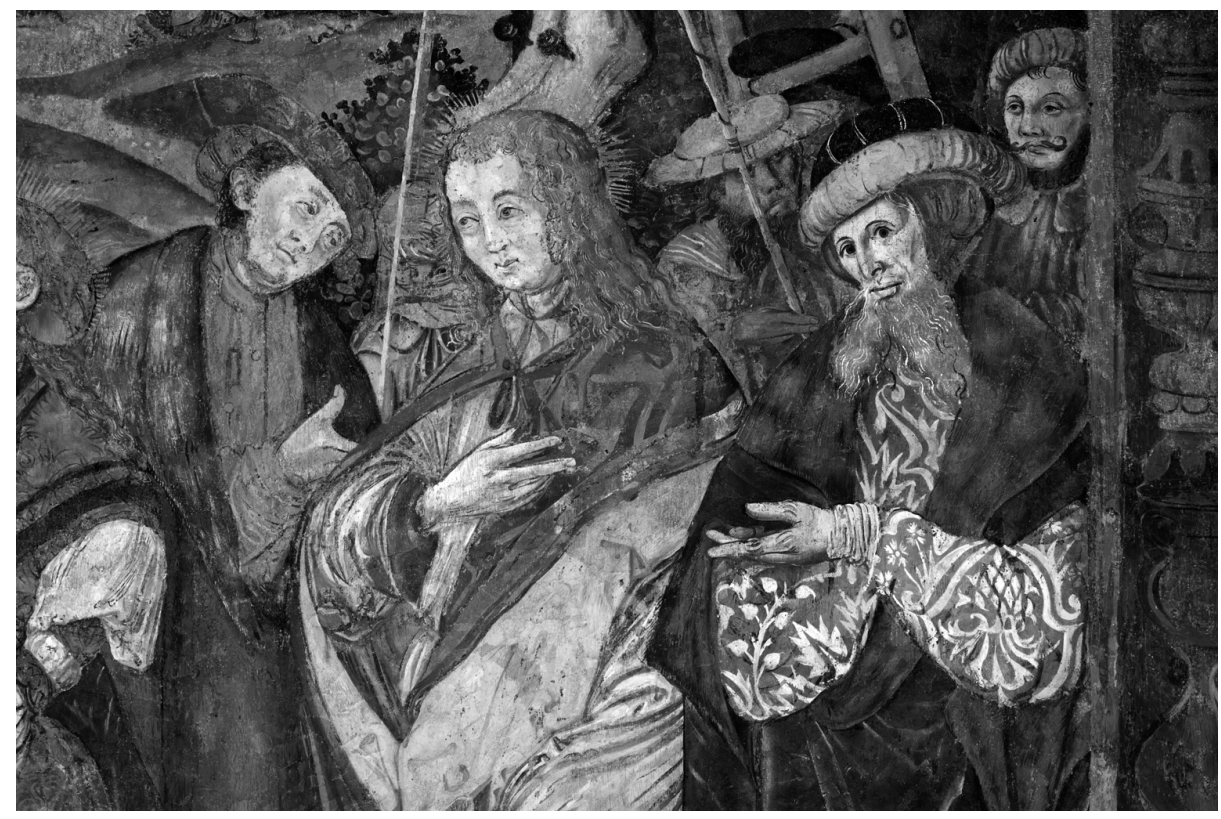

Abb. 2: Johannes Hassensteiner von Lobkowicz als Josef von Arimathia (rechts) mit dem hl. Johannes, Wandmalerei im Presbyterium der Kirche der Vierzehn heiligen Nothelfer in Kaaden (Kadaň), um 1530.

Die Herren von Plauen, d. h. die Burggrafen von Meißen, mit denen Jaroslaus Hassensteiner von Lobkowicz verwandt war, gehörten zu den bedeutendsten Akteuren des albertinischen Sachsen. Der sächsische Herzog Georg der Bärtige lehnte aus kirchenpolitischen sowie theologischen Gründen die Postulate der Wittenberger Reformation $a b$, zugleich aber forderte er zur allgemeinen Kirchenreform auf. ${ }^{12}$ Die sächsisch-albertinischen Eliten blieben bis zum Tod Georgs 1539 dem konzilianten Erasmianismus treu. Auch Margaretha von Plauen gehörte zu einer erasmianisch gesinnten Familie, und in diesem Geist wirkte sie in der Familie von Lobkowicz. Das Ergebnis war jedoch, dass ihre Söhne Heinrich und Sebastian zur böhmischen Utraquistenkirche, respektive zum Luthertum, konvertierten. Die christologisch verankerten Wandmalereien im Presbyterium der Franziskanerkirche der Vierzehn heiligen Nothelfer in Kaaden sind eigentlich ein symbolischer Beleg jener Übergangszeit, als weder der tridentinische Katholizismus noch die lutherische Orthodoxie schon existierten. Im Milieu der mitteleuropäischen Eliten bestanden daneben noch die reformkonzilianten Gruppen, die ihre Inspiration eher bei Erasmus oder Melanchthon such-

11), Kiel 2009. Vgl. Bernd Moeller, Annaberg als Stadt der Reformation, in: Harald Marx/Cecilie Hollberg (Hg.), Glaube und Macht. Sachsen im Europa der Reformationszeit, Dresden 2004, S. 103-111; Petr HLAVÁČEK, The Constants of Spirituality and Ecclesiastical Politics in the Family of the Bohemian King George of Poděbrady and of the Princes of Münsterberg, in: The Bohemian Reformation and Religious Practice 9 (2014), S. 185-197.

12 Vgl. Christoph Volkmar, Reform statt Reformation. Die Kirchenpolitik Herzog Georgs von Sachsen 1488-1525 (Spätmittelalter, Humanismus, Reformation. Studies in the Late Middle Ages, Humanism and the Reformation 41), Tübingen 2008. 
ten. Und zu ihnen gehörten zwischen 1520 und 1540 gerade die Hassensteiner von Lobkowicz. Die Kaadner Wandmalereien, die Tafelbilder imitieren, schuf jemand aus der Wittenberger Cranach-Werkstatt, die damals sowohl für lutherische als auch katholische Auftraggeber arbeitete. ${ }^{13}$

Es ist höchst symbolisch, dass gerade diese so spezifischen Kaadner Wandmalereien zu Zeugen des böhmisch-sächsischen Versuchs eines Religionsfriedens nicht nur in Böhmen und Sachsen, sondern im ganzen Heiligen Römischen Reich wurden. Im Juni 1534 wurde in Kaaden der sog. Kaadner Frieden oder Vertrag zwischen den katholischen und lutherischen Fürsten abgeschlossen. Die Vorbereitungsphase fand im Franziskanerkloster in der sächsischen Bergstadt Annaberg statt, die Hauptverhandlungen und heftigen Disputationen wurden in Kaaden vorangetrieben. Im Garten des Kaadner Franziskanerklosters fand dann ein Versöhnungsmahl der Hauptakteure dieses katholisch-lutherischen Gipfeltreffens statt. Die politischen Akteure, die verschiedene kirchenpolitische sowie spirituelle Strömungen repräsentierten, feierten in der Franziskanerkirche eine gemeinsame Liturgie, und sie besichtigten auch die hiesigen Wandmalereien. Durch die Kirche promenierten zum Beispiel der römische und böhmische König Ferdinand I. von Habsburg mit seinem Diplomaten Siegmund von Herberstein, daneben der sächsische Kurfürst Johann Friedrich mit Georg Spalatin, weiter Kardinal Albrecht von Brandenburg und der sächsische Herzog Georg der Bärtige. Es fehlte auch der päpstliche Nuntius Pier Paolo Vergerio nicht, der sich später der Reformation anschloss, oder die böhmische katholische und utraquistische Aristokratie.

Wie wurden die Wandmalereien von dieser heterogenen Gruppe eigentlich reflektiert? Wir wissen nur, dass alle ohne Unterschied den Kaadner Franziskanern Almosen spendeten. Sicher muss das eine ungewöhnliche Erscheinung gewesen sein, als sich in der Kirche mit der lobkowiczschen Begräbnisstätte Kardinal Albrecht und Nuntius Vergerio öffentlich in Anwesenheit des Kurfürsten Johann Friedrich beschwerten, dass es im böhmischen Kaaden ähnlich wie im „ketzerischen“ Sachsen wäre, denn hier lebten außer den Katholiken und Utraquisten schon die verfluchten „lutherani bestiali“. Die Familie von Lobkowicz war damals schon nicht mehr der Herrscher Kaadens, da die Stadt sich mit Hilfe anderer böhmischer königlicher Städte aus der lobkowiczschen Verpfändung auslöste. ${ }^{14}$

Die damalige böhmisch-sächsische Kulturlandschaft, deren Herz das Erzgebirge bildete, erlebte ihren Höhepunkt gerade während des Reformationsjahrhunderts, als diese Region durch den Bergbauboom und eine unerwartet tolerante Multikonfessionalität die Eliten ganz Mitteleuropas anzog. An dieser dynamischen und mannigfal-

13 Vgl. Magdaléna Hamsíková, Cranach und Böhmen. Die sächsische höfische und kirchliche Repräsentation im Spiegel der Malerei der ersten Hälfte des 16. Jahrhunderts in Böhmen, in:Eva Doležalová/RobertŠimůnek(Hg.), Ecclesia als Kommunikationsraum in Mitteleuropa (13.-16. Jahrhundert) (Veröffentlichungen des Collegium Carolinum 122), München 2011, S. 339-360; Aleš Mudra/Michaela OtTová, Trans montes. Podoby středověkého umění v severozápadních Čechách [Trans montes. Die Formen der mittelalterlichen Kunst in Nordwestböhmen], Praha 2014, S. 93-107, 143-166; PETR HLAvÁČEK, Lutheran Culture in Bohemia, in: Kateřina Horníčková/Michal Šroněk (Hg.), From Hus to Luther. Visual Culture in the Bohemian Reformation (1380-1620), Turnhout 2016, S. 165-192.

14 Petr HlaváČEK, Kadaňský mír z roku 1534. Katolicko-luterský pokus o záchranu jednoty Svaté říše římské [Der Frieden von Kaaden 1534. Ein katholisch-evangelischer Versuch um die Rettung der Einheit des Heiligen Römischen Reiches], in: David Jurech (Hg.), Lutheranus 2007/2008, Band 2, Praha 2008, S. 138-146. 


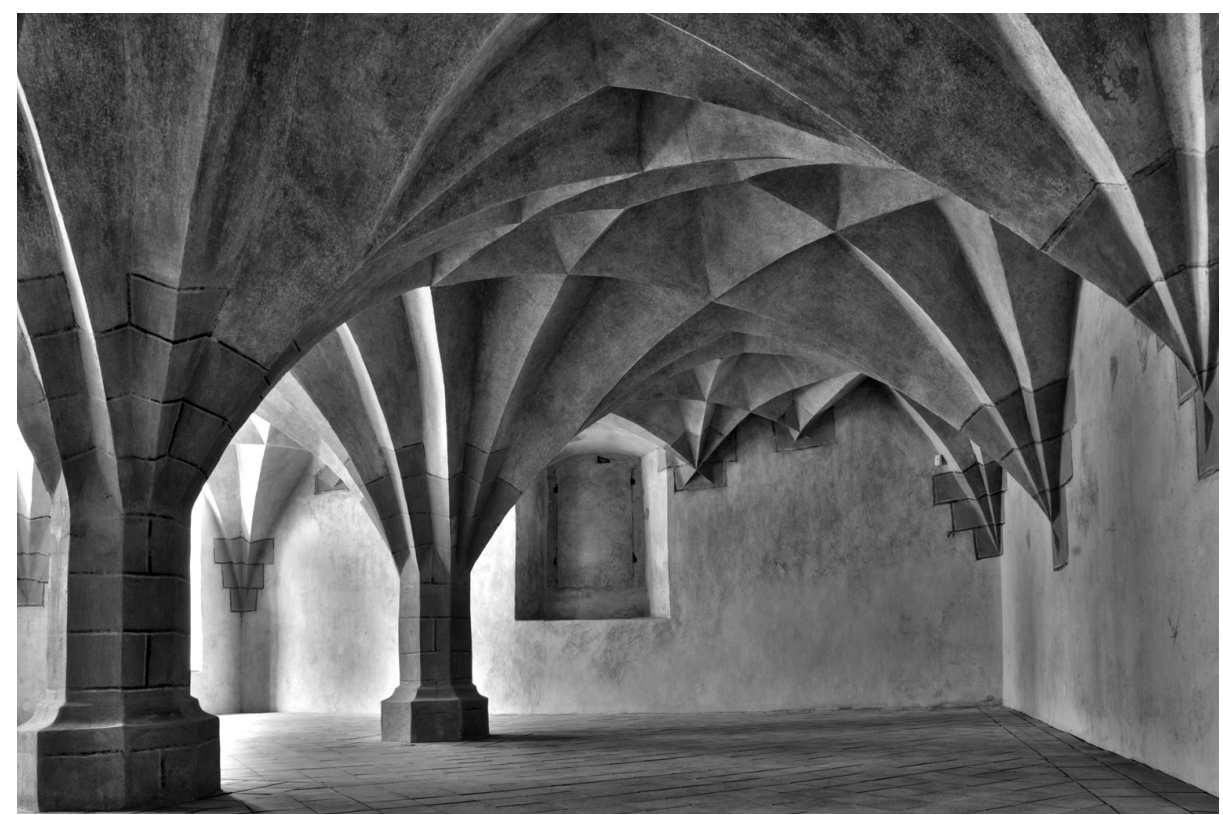

Abb. 3: Sog. Coenaculum mit Zellengewölben im Franziskanerkloster in Kaaden (Kadaň), vor 1500.

tigen Interaktivität hatte auch die aristokratische Familie der Hassensteiner von Lobkowicz Anteil, denn ihre Mitglieder sprachen Tschechisch und Deutsch, in ihrem intellektuellen Umkreis pflegte man nicht nur Latein, sondern auch Griechisch und sogar Hebräisch. Eine wichtige Rolle in dieser spezifischen Wesensart der Hassensteiner von Lobkowicz spielte auch, dass sich schon Johannes und sein Bruder Bohuslaus nicht nur als Tschechen, sondern auch als Europäer fühlten. Für viele ihrer Zeitgenossen war das wahrscheinlich etwas Unverständliches, für andere bildete das selbstbewusste Europäertum und die Zugehörigkeit zu Europa (nicht nur zur „Christenheit“) einen Teil ihrer persönlichen Identität. Was waren die Hauptgründe für diese lobkowiczsche Mentalität der Offenheit? Sicher auch die Tatsache, dass gerade die größten Persönlichkeiten der Familie aus ihrer engen Heimat im böhmisch-sächsischen Erzgebirge heraustraten, sie bewegten sich auch außerhalb Europas, nämlich in Asien und Afrika, in ihren Korrespondenzen träumten sie von der amerikanischen Neuen Welt. Also: „Bohemia“ oder „Saxonia“? Schon an der Wende vom Mittelalter zur Neuzeit hielten manche Mitteleuropäer, die Angehörigen der sog. Eliten, nicht nur „Europa“, sondern auch „totus mundus“ für ihre Heimat. ${ }^{15}$

15 Petr Hlaváčen, Christianity, Europe, and (Utraquist) Bohemia: The Theological and Geographic Concepts in the Middle Ages and Early Modern Times, in: Zdeněk V. David/David R. Holeton (Hg.), The Bohemian Reformation and Religious Practice 7 (2009), S. 19-41; Ders., „Respublica Christiana“ aneb spiritualita a církevně-politické představy Bohuslava Hasištejnského z Lobkowicz [„Respublica Christiana“ oder die Spiritualität und die kirchenpolitischen Vorstellungen von Bohuslaus Hassensteiner von Lobkowicz], in: Marta Vaculínová (Hg.), Sborník Národního muzea v Praze (Řada C - literární historie 52), Num. 1-4, Praha 2007, S. 5-7. 